\title{
Article
}

\section{Existence result for a semipositone fractional boundary value problem}

\author{
Șerife Müge Ege ${ }^{1, *}$ and Fatma Serap Topal ${ }^{1}$ \\ 1 Department of Mathematics, Ege University, Bornova, Izmir 35100 Turkey. \\ * Correspondence: f.serap.topal@ege.edu.tr \\ Academic Editor: Ghaus Ur Rahman \\ Received: 15 June 2021; Accepted: 10 October 2021; Published: 1 November 2021.
}

\begin{abstract}
This work deals with a boundary value problem for a nonlinear semipositone multi-point fractional differential equation. By using the Schauder fixed point theorem, we show the existence of one solution for this problem. Our result extend some recent works in the literature.
\end{abstract}

Keywords: Caputo fractional derivative; Boundary value problem; Changing sign nonlinearity; Fixed point theorem.

MSC: 34B10, 34B15, 26A33.

\section{Introduction}

$\mathbf{F}$ ractional calculus is the extension of integer derivatives to real or complex number derivatives. Therefore, it has gained an important place in terms of examining the sensitivity of many phenomena in natural sciences. Most of the problems in scientific fields such as physics, biology, astronomy, medical sciences, optical fibers, chemical biology, radiology can be modeled by differential equations [1-6]. The fractional orders of the differential equations enable the investigations to be more comprehensive and obtain optimal solutions. That's why fractional differential equations have been the focus of various researchers over the past decades. Articles and books have been written to demonstrate the existence of solutions to such equations or to develop new solution methods [7-9].

Many people have studied the existence and positive solutions or multiplicity of solutions for nonlinear fractional boundary value problems using fixed point theorems such as the Schauder fixed-point theorem, the Guo-Krasnosel'skii fixed-point theorem, and the Leggett-Williams fixed-point theorem. In recent studies, boundary value problems involving multi point boundary condition and fractional differential equations have attracted attention.

In order to guarantee the existence of positive solutions for boundary value problems, the nonlinearity is usually nonnegative. If the nonlinearity changing sign, it will bring much more difficulties to the study of the problem. Because of this, there is only a few study on such problems [10-14].

In [15], Ahmad consider the two point Liouville-Caputo boundary value problem

$$
\begin{array}{ll}
{ }^{c} D^{\beta} x(t)=-f(t, x(t)), & t \in(a, b) \\
x(a)=\delta_{1}, \quad x(b)=\delta_{2}, & \delta_{i} \in \mathbb{R}, i=1,2
\end{array}
$$

where ${ }^{c} D^{\beta}$ denotes the Caputo fractional derivative of order $\beta$ with $1<\beta<2$ and $f$ is a continuous function.

In this paper, we investigate the existence result for the following m-point fractional boundary value problem with changing sign nonlinearity,

$$
D_{a}^{\beta} u(t)+f(t, u(t))=0, \quad t \in(a, b)
$$




$$
u(a)=\delta_{1}, \quad u(b)=\sum_{i=1}^{m-2} \xi_{i} u\left(\eta_{i}\right)+\delta_{2}, \quad \delta_{i} \in \mathbb{R}, i=1,2,
$$

where $D_{a}^{\beta}$ is the standard Caputo fractional derivatives of fractional order $\beta$ with $1<\beta \leq 2, \xi_{i} \quad(1 \leq i \leq$ $m-2)$ are positive real constants with $0<\sum_{i=1}^{m-2} \xi_{i}<1, \eta_{i} \in(a, b)$ and a continuous function $f:[a, b] \times \mathbb{R} \rightarrow \mathbb{R}$ may change sign.

The paper is organized as follows: In Section 2, present some background materials and preliminaries. In Section 3, we give an existence result and also an example is given to exemplify main result.

\section{Preliminaries}

In this section we gather some preliminary definitions, theorems and fundamental results of fractional calculus theory that will be used in subsequent section. Basic properties of the fractional calculus and its applications can be found in Kilbas, Srivastava and Trujillo [4] and Podlubny [6].

Definition 1. For a function $f$ given on the interval $[a, b]$, the Caputo derivative of fractional order $r$ is defined as

$$
D^{r} f(t)=\frac{1}{\Gamma(n-r)} \int_{0}^{t}(t-s)^{n-r-1} f^{(n)}(s) d s, \quad n=[r]+1,
$$

where $[r]$ denotes the integer part of $r$.

Definition 2. The Riemann-Liouville fractional integral of order $r$ for a function $f$ is defined as

$$
I^{r} f(t)=\frac{1}{\Gamma(r)} \int_{0}^{t}(t-s)^{r-1} f(s) d s, \quad r>0,
$$

where $[r]$ denotes the integer part of $r$.

Lemma 1. Let $r>0$. Then the differential equation $D^{r} x(t)=0$ has solutions

$$
x(t)=c_{0}+c_{1} t+c_{2} t^{2}+\cdots+c_{n-1} t^{n-1},
$$

where $c_{i} \in \mathbb{R}, i=0,1,2, \ldots, n, n=[r]+1$.

Lemma 2. Let $r>0$. Then

$$
I^{r}\left(D^{r} x\right)(t)=x(t)+c_{0}+c_{1} t+c_{2} t^{2}+\ldots+c_{n-1} t^{n-1},
$$

where $c_{i} \in \mathbb{R}, i=0,1,2, \ldots, n, n=[r]+1$.

For finding a solution of the problem (1)-(2), we first consider the following fractional differential equation

$$
-D_{a}^{\beta} u(t)=h(t)
$$

with the boundary condition (2).

Let we define $\Delta:=b-a-\sum_{i=1}^{m-2} \xi_{i}\left(\eta_{i}-a\right)$.

Lemma 3. Let $\beta \in(1,2]$ and $t \in[a, b]$. The boundary value problem (3)-(2) has a unique solution $u$ in the form

$$
u(t)=\left[1+\frac{\sum_{i=1}^{m-2} \xi_{i}-1}{\Delta}(t-a)\right] \delta_{1}+\frac{t-a}{\Delta} \delta_{2}+\int_{a}^{b} G(t, s) h(s) d s,
$$


where

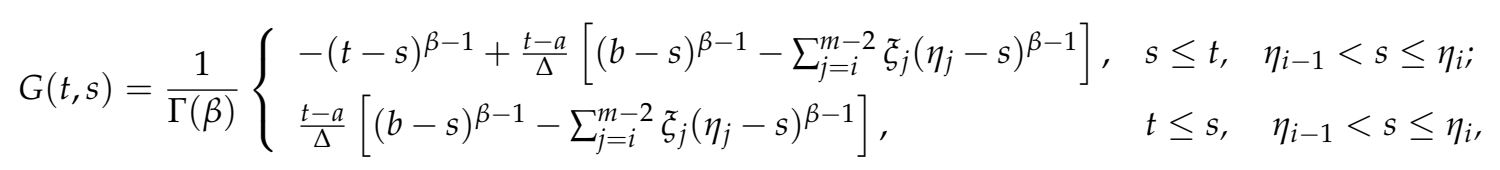

$i=1,2, \ldots m-2$.

Proof. The equation $D^{\beta} u(t)=-h(t)$ has a unique solution

$$
u(t)=-\frac{1}{\Gamma(\beta)} \int_{a}^{t}(t-s)^{\beta-1} h(s) d s+c_{0}+c_{1}(t-a),
$$

where $c_{0}, c_{1} \in \mathbb{R}$.

$$
\begin{aligned}
& \text { By } u(a)=\delta_{1} \text { and } u(b)=\sum_{i=1}^{m-2} \xi u\left(\eta_{i}\right)+\delta_{2}, \text { we have } c_{0}=\delta_{1} \text { and } \\
& c_{1}=\frac{1}{\Delta}\left(-\frac{1}{\Gamma(\beta)} \sum_{i=1}^{m-2} \xi_{i} \int_{a}^{\eta_{i}}\left(\eta_{i}-s\right)^{\beta-1} h(s) d s+\frac{1}{\Gamma(\beta)} \int_{a}^{b}(b-s)^{\beta-1} h(s) d s+\delta_{1}\left[\sum_{i=1}^{m-2} \xi_{i}-1\right]+\delta_{2}\right) .
\end{aligned}
$$

Substituting $c_{0}, c_{1}$ into equation (5) we find,

$$
\begin{aligned}
u(t)= & {\left[1+\frac{\sum_{i=1}^{m-2} \xi_{i}-1}{\Delta}(t-a)\right] \delta_{1}+\frac{t-a}{\Delta} \delta_{2}-\frac{1}{\Gamma(\beta)}\left(\int_{a}^{t}(t-s)^{\beta-1} h(s) d s+\frac{t-a}{\Delta} \sum_{i=1}^{m-2} \xi_{i} \int_{a}^{\eta_{i}}\left(\eta_{i}-s\right)^{\beta-1} h(s) d s\right.} \\
& \left.-\frac{t-a}{\Delta} \int_{a}^{b}(b-s)^{\beta-1} h(s) d s\right) \\
= & {\left[1+\frac{\sum_{i=1}^{m-2} \xi_{i}-1}{\Delta}(t-a)\right] \delta_{1}+\frac{t-a}{\Delta} \delta_{2}+\int_{a}^{b} G(t, s) h(s) d s, }
\end{aligned}
$$

where $G(t, s)$ is defined by (4). The proof is completed.

Lemma 4. If $0<\sum_{i=1}^{m-2} \xi_{i}<1$, then
i) $\Delta>0$,
ii) $(b-s)^{\beta-1}-\sum_{j=i}^{m-2} \xi_{j}\left(\eta_{j}-s\right)^{\beta-1}>0$.

Proof. i) We can easily see that

$$
\begin{aligned}
& \eta_{i}<b, \\
& \xi_{i}\left(\eta_{i}-a\right)<\xi_{i}(b-a), \\
& -\sum_{i=1}^{m-2} \xi_{i}\left(\eta_{i}-a\right)>-\sum_{i=1}^{m-2} \xi_{i}(b-a), \\
& b-a-\sum_{i=1}^{m-2} \xi_{i}\left(\eta_{i}-a\right)>b-a-\sum_{i=1}^{m-2} \xi_{i}(b-a)=(b-a)\left[1-\sum_{i=1}^{m-2} \xi_{i}\right] .
\end{aligned}
$$

If $1-\sum_{i=1}^{m-2} \xi_{i}>0$, then $b-a-\sum_{i=1}^{m-2} \xi_{i}\left(\eta_{i}-a\right)>0$. So we have $\Delta>0$.

ii) Since $0<\beta-1 \leq 1$, we have $b-a>b-s>(b-s)^{\beta-1}$ and $\eta_{i}-a>\eta_{i}-s>\left(\eta_{i}-s\right)^{\beta-1}$. Also we know that $\left(\eta_{j}-s\right)^{\beta-1}<(b-s)^{\beta-1}$. Thus we have

$$
\sum_{j=i}^{m-2} \xi_{j}\left(\eta_{j}-s\right)^{\beta-1}<\sum_{j=i}^{m-2} \xi_{j}(b-s)^{\beta-1} \leq(b-s)^{\beta-1} \sum_{i=1}^{m-2} \xi_{i}<(b-s)^{\beta-1}
$$


and so $(b-s)^{\beta-1}-\sum_{j=i}^{m-2} \xi_{j}\left(\eta_{j}-s\right)^{\beta-1}>0$.

Remark 1. Let $G(t, s)$ be the Green's function for the problem (1)-(2). It is easy to find that

$$
\begin{aligned}
\int_{a}^{b}|G(t, s)| d s & \leq \frac{1}{\Gamma(\beta)} \int_{a}^{t}(t-s)^{\beta-1} d s+\frac{t-a}{\Gamma(\beta) \Delta} \sum_{i=1}^{m-2} \xi_{i} \int_{a}^{\eta_{i}}\left(\eta_{i}-s\right)^{\beta-1} d s+\frac{t-a}{\Delta \Gamma(\beta)} \int_{a}^{b}(b-s)^{\beta-1} d s \\
& =\frac{(t-a)^{\beta}}{\Gamma(\beta+1)}+\frac{t-a}{\Delta \Gamma(\beta+1)} \sum_{i=1}^{m-2} \xi_{i}\left(\eta_{i}-a\right)^{\beta}+\frac{t-a}{\Delta \Gamma(\beta+1)}(b-a)^{\beta} \\
& \leq \frac{(b-a)^{\beta}}{\Gamma(\beta+1)}+\frac{b-a}{\Delta \Gamma(\beta+1)} \sum_{i=1}^{m-2} \xi_{i}\left(\eta_{i}-a\right)^{\beta}+\frac{(b-a)^{\beta+1}}{\Delta \Gamma(\beta+1)}:=M .
\end{aligned}
$$

Remark 2. Let $p(t) \in L^{1}[a, b]$ and $w(t)$ be a solution of the problem

$$
\left\{\begin{array}{l}
D_{a}^{\beta} u(t)+p(t)=0 \\
u(a)=0, \quad u(b)=\sum_{i=1}^{m-2} \xi_{i} u\left(\eta_{i}\right)
\end{array}\right.
$$

then $w(t)=\int_{a}^{b} G(t, s) p(s) d s$.

The following fixed point theorem is fundamental and important to the proof of our main result.

Theorem 1. [14][Schauder-Tychonoff Fixed Point Theorem] Let X be a Banach space. Assume that $K$ is a closed, bounded, convex subset of $X$. If $T: K \longrightarrow K$ is compact, then $T$ has a fixed point in $K$.

\section{Existence Result}

We also make the following assumption throughout this paper.

(H1) There exists a nonnegative function $p \in L^{1}[a, b]$ and $\int_{a}^{b} p(t) d t>0$ such that $f(t, u) \geq-p(t)$ for all $(t, u) \in[a, b] \times \mathbb{R}$

(H2) $f(t, u) \neq 0$, for $(t, u) \in[a, b] \times \mathbb{R}$.

Let $B=\mathbb{C}([a, b], \mathbb{R})$ denote the Banach space of all continuous function from $[a, b]$ into $\mathbb{R}$ endowed with the usual norm $\|u\|=\sup \{|u(t)|: t \in[a, b]\}$.

First we shall show that the following fractional equation

$$
D_{a}^{\beta} u(t)+F\left(t, u^{*}(t)\right)=0, \quad t \in[a, b]
$$

with the boundary condition (2) has a solution, where $F:[a, b] \times \mathbb{R} \rightarrow \mathbb{R}$

$$
F(t, z)= \begin{cases}f(t, z)+p(t), & z \geq 0 \\ f(t, 0)+p(t), & z \leq 0\end{cases}
$$

and $u^{*}(t)=\max \{(u-w)(t), 0\}$ such that $w$ is the unique solution of the problem (7). We define an operator $T: B \rightarrow B$ associated with the problem (8)-(2) as

$$
(T u)(t)=\left[1+\frac{\sum_{i=1}^{m-2} \xi_{i}-1}{\Delta}(t-a)\right] \delta_{1}+\frac{t-a}{\Delta} \delta_{2}+\int_{a}^{b} G(t, s) F\left(s, u^{*}(s)\right) d s
$$

where $G(t, s)$ is given by (4). The existence of a fixed point for the operator $T$ implies the existence of a solution for the problem (8)-(2). 
Theorem 2. Assume that (H1)-(H2) are satisfied. If $K>0$ satisfies $\left[1+\frac{\sum_{i=1}^{m-2} \xi_{i}-1}{\Delta}(b-a)\right] \delta_{1}+\frac{b-a}{\Delta} \delta_{2}+L M \leq K$ where $L \geq \max \{|F(t, u)|: t \in[a, b],|u| \leq K\}$ and $M$ is given in (6) then the problem (8)-(9) has a solution $u(t)$.

Proof. Let we define $P:=\{u \in B:\|u\| \leq K\}$. It can be easily seen that $P$ is a closed, bounded and convex subset of $B$ to which Schauder fixed point theorem is applicable. Define $T: P \longrightarrow B$ by (10). It is easily seen that $T: P \longrightarrow B$ is continuous. Claim $T: P \longrightarrow P$. Let $u \in P$. Consider $u^{*}(t) \leq u(t) \leq K$ for all $t \in[a, b]$. Then

$$
\begin{aligned}
|T u(t)| & =\left|\left[1+\frac{\sum_{i=1}^{m-2} \xi_{i}-1}{\Delta}(t-a)\right] \delta_{1}+\frac{t-a}{\Delta} \delta_{2}+\int_{a}^{b} G(t, s) F\left(s, u^{*}(s)\right) d s\right| \\
& \leq\left[1+\frac{\sum_{i=1}^{m-2} \xi_{i}-1}{\Delta}(b-a)\right] \delta_{1}+\frac{b-a}{\Delta} \delta_{2}+L M \leq K
\end{aligned}
$$

for all $t \in[a, b]$. This implies that $\|T u\| \leq K$. Hence $T: K \longrightarrow K$. Using the Arzela-Ascoli theorem it can be shown that $T: K \longrightarrow K$ is a compact operator. Hence $T$ has a fixed point $u$ in $P$ by the Schauder-Tychonov theorem. This implies that $u$ is a solution of the problem (8)-(2).

Lemma 5. $u^{*}(t)$ is the solution of the boundary value problem (1)-(2) with $u(t)>w(t)$ for all $t \in[a, b]$ if and only if $u=u^{*}+w$ is the positive solution of the boundary value problem (8)-(2).

Proof. Let $u(t)$ is the solution of the boundary value problem (8)-(2). Then

$$
\begin{aligned}
u(t) & =\left[1+\frac{\sum_{i=1}^{m-2} \xi_{i}-1}{\Delta}(t-a)\right] \delta_{1}+\frac{t-a}{\Delta} \delta_{2}+\frac{1}{\Gamma(\beta)} \int_{a}^{b} G(t, s) F\left(s, u^{*}(s)\right) d s \\
& =\left[1+\frac{\sum_{i=1}^{m-2} \xi_{i}-1}{\Delta}(t-a)\right] \delta_{1}+\frac{t-a}{\Delta} \delta_{2}+\frac{1}{\Gamma(\beta)} \int_{a}^{b} G(t, s)\left(f\left(s, u^{*}(s)\right)+p(s)\right) d s \\
& =\left[1+\frac{\sum_{i=1}^{m-2} \xi_{i}-1}{\Delta}(t-a)\right] \delta_{1}+\frac{t-a}{\Delta} \delta_{2}+\frac{1}{\Gamma(\beta)} \int_{a}^{b} G(t, s) f(s,(u-w)(s)) d s+\frac{1}{\Gamma(\beta)} \int_{a}^{b} G(t, s) p(s) d s \\
& =\left[1+\frac{\sum_{i=1}^{m-2} \xi_{i}-1}{\Delta}(t-a)\right] \delta_{1}+\frac{t-a}{\Delta} \delta_{2}+\frac{1}{\Gamma(\beta)} \int_{a}^{b} G(t, s) f(s,(u-w)(s)) d s+w(t)
\end{aligned}
$$

or

$$
u(t)-w(t)=\left[1+\frac{\sum_{i=1}^{m-2} \xi_{i}-1}{\Delta}(t-a)\right] \delta_{1}+\frac{t-a}{\Delta} \delta_{2}+\frac{1}{\Gamma(\beta)} \int_{a}^{b} G(t, s) f(s,(u-w)(s)) d s
$$

and hence we get

$$
u^{*}(t)=\left[1+\frac{\sum_{i=1}^{m-2} \xi_{i}-1}{\Delta}(t-a)\right] \delta_{1}+\frac{t-a}{\Delta} \delta_{2}+\frac{1}{\Gamma(\beta)} \int_{a}^{b} G(t, s) f\left(s, u^{*}(s)\right) d s .
$$

In other words, if $u^{*}$ is a solution of the boundary value problem (1)-(2) then we get

$$
D_{a}^{\beta}\left(u^{*}(t)+w(t)\right)=D_{a}^{\beta} u^{*}(t)+D_{a}^{\beta} w(t)=-f\left(t, u^{*}(t)\right)-p(t)=-\left[f\left(t, u^{*}(t)\right)+p(t)\right]=-F\left(t, u^{*}(t)\right),
$$

which implies that

$$
D_{a}^{\beta} u(t)=-F\left(t, u^{*}(t)\right) .
$$

Also from the boundary conditions, we easily see that

$$
u^{*}(a)=u(a)-w(a)=u(a)-0=\delta_{1},
$$


i.e., $u(0)=\delta_{1}$ and

$$
\begin{aligned}
& u^{*}(b)=\sum_{i=1}^{m-2} \xi_{i} u^{*}\left(\eta_{i}\right)+\delta_{2} \\
& u(b)-w(b)=\sum_{i=1}^{m-2} \xi_{i}\left(u\left(\eta_{i}\right)-w\left(\eta_{i}\right)\right)+\delta_{2}=\sum_{i=1}^{m-2} \xi_{i} u\left(\eta_{i}\right)-\sum_{i=1}^{m-2} \xi_{i} w\left(\eta_{i}\right)+\delta_{2},
\end{aligned}
$$

i.e.,

$$
u(b)=\sum_{i=1}^{m-2} \xi_{i} u\left(\eta_{i}\right)+\delta_{2} .
$$

Therefore $u(t)$ is a solution of the boundary value problem (8)-(9).

Example 1. Consider the following fractional boundary value problem

$$
\begin{gathered}
D^{\frac{4}{3}} u(t)+f(t, u(t))=0, \quad t \in(0,1), \\
u(0)=1, \quad u(1)=\frac{1}{4} u\left(\frac{1}{2}\right)+\frac{1}{2} u\left(\frac{1}{4}\right)+\frac{1}{8} u\left(\frac{1}{5}\right)-1
\end{gathered}
$$

with the function $f(t, u(t))=\frac{e^{t}}{1+t^{2}} \sin (u(t))$.

Choosing $p(t)=e^{t}$ we get $\int_{0}^{1} e^{t} d t=e-1>0$, so it is easy to check that the assumptions (H1)-(H2) hold. Calculating $\Delta=\frac{29}{40}, M \cong 2.9$ and seeing $|F(t, u)|<2 e=L$ such that $|u| \leq K$ where $K=20$, we can easily verify that

$$
\left[1+\frac{\sum_{i=1}^{m-2} \xi_{i}-1}{\Delta}(b-a)\right] \delta_{1}+\frac{b-a}{\Delta} \delta_{2}+L M \cong 15,3 \leq 20 .
$$

Then applying Theorem 2 the problem (11)-(12) has a solution $u(t)$.

Author Contributions: All authors contributed equally to the writing of this paper. All authors read and approved the final manuscript.

Conflicts of Interest: "The authors declare no conflict of interest."

\section{References}

[1] Ahmad, B. (2010). Existence of solutions for irregular boundary value problems of nonlinear fractional differential equations. Applied Mathematics Letters, 23(4), 390-394.

[2] Bai, Z. (2010). On positive solutions of a nonlocal fractional boundary value problem. Nonlinear Analysis: Theory, Methods $\mathcal{E}$ Applications, 72(2), 916-924.

[3] Bai, Z., \& Lü, H. (2005). Positive solutions for boundary value problem of nonlinear fractional differential equation. Journal of Mathematical Analysis and Applications, 311(2), 495-505.

[4] Kilbas, A. A., Srivastava, H. M., \& Trujillo, J. J. (2006). Theory and Applications of Fractional Differential Equations (Vol. 204). elsevier.

[5] Miller, K. S., \& Ross, B. (1993). An Introduction to the Fractional Calculus and Fractional Differential Equations. Wiley, New York.

[6] Podlubny, I. (1999). Fractional Differential Equations, Mathematics in Science and Engineering. Academic Press New York.

[7] Liang, S., \& Zhang, J. (2009). Positive solutions for boundary value problems of nonlinear fractional differential equation. Nonlinear Analysis: Theory, Methods \& Applications, 71(11), 5545-5550.

[8] Zhang, S. (2006). Positive solutions for boundary-value problems of nonlinear fractional differential equations. Electronic Journal of Differential Equations, 36(2), 1-12.

[9] Zhong, W., \& Lin, W. (2010). Nonlocal and multiple-point boundary value problem for fractional differential equations. Computers \& Mathematics with Applications, 59(3), 1345-1351.

[10] Li, F., Zhang, Y., \& Li, Y. (2008). Sign-changing solutions on a kind of fourth-order Neumann boundary value problem. Journal of Mathematical Analysis and Applications, 344(1), 417-428.

[11] Li, Y., \& Li, F. (2008). Sign-changing solutions to second-order integral boundary value problems. Nonlinear Analysis: Theory, Methods E Applications, 69(4), 1179-1187. 
[12] Liu, Z., Ding, Y., Liu, C., \& Zhao, C. (2020). Existence and uniqueness of solutions for singular fractional differential equation boundary value problem with p-Laplacian. Advances in Difference Equations, 2020, Article No. 83. https://doi.org/10.1186/s13662-019-2482-9.

[13] Xu, X. (2004). Multiple sign-changing solutions for some m-point boundary-value problems. Electronic Journal of Differential Equations, 89, 1-14.

[14] Tychonoff, A. (1935). Ein fixpunktsatz. Mathematische Annalen, 111(1), 767-776.

[15] Ahmad, B. (2017). Sharp estimates for the unique solution of two-point fractional-order boundary value problems. Applied Mathematics Letters, 65, 77-82.

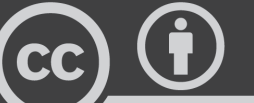

BY

(C) 2021 by the authors; licensee PSRP, Lahore, Pakistan. This article is an open access article distributed under the terms and conditions of the Creative Commons Attribution (CC-BY) license (http://creativecommons.org/licenses/by/4.0/). 